\title{
Is there is Need for Ubiquinone (CoQ10) Supplementation in Statin- Associated Myopathy?
}

\author{
Dasa Skripova $^{1, *}$, Pella Daniel ${ }^{1}$, Kozlikova Katarina $^{2}$ and Rybar Rafael ${ }^{1}$

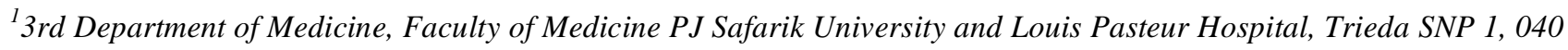 \\ 66 Kosice, Slovakia \\ ${ }^{2}$ Institute of Medical Physics, Biophysics, Informatics and Telemedicine, Faculty of Medicine, Comenius University \\ Bratislava, Sasinkova 2, 81108 Bratislava, Slovakia
}

\begin{abstract}
Statins are currently the most effective drugs in reducing low-density lipoprotein cholesterol (LDL-C). With their mechanism of action, by inhibiting 3-hydroxy-3 methylglutharyl coenzyme A reductase, statins decrease cholesterol production. The same biosynthetic pathway is shared by ubiquinone or coenzyme Q10. Statins block production of dekaprenyl-4-benzoate, a precursor of coenzyme Q10 (CoQ10), which is an essential component in mitochondrial transport system. Ubiquinone deficiency may affect oxidative phosphorylation and adenosine triphosphate production, which can subsequently result in impairing of muscle energy metabolism and contribute to development of myopathy. Statin therapy also decreases antioxidant status in the body, resulting in to increase in free radical damage to cells in various parts of body; liver, nerves, muscles. Statins can decrease natural antioxidant protection present in our body and predispose toxicity.

Statin-associated myopathy is the most common side effect of statin treatment often lead to statin dose reduction, or therapy cessation, which can negatively affect cardiovascular risk management. The spectrum of statin-related myopathy ranges from common but clinically benign myalgia to rare but life-threatening rhabdomyolysis. Observational studies suggest that myalgia can occur in up to $10 \%$ of persons prescribed statins, whereas rhabdomyolysis continues to be rare.

Statins lower circulating levels of CoQ10 up to 54\%, whereas several studies did not confirmed a lowering of CoQ10 levels in muscles during statin therapy and the evidence was given also on low dose of statin therapy, which did not appear to reduce intramuscular levels of CoQ10 in symptomatic patients with statin myopathy. The conflicting results have been published on the impaired mitochondrial function, which was found during the analysis of the myocyte cells in patients treated with statins. The supplementation of CoQ10 results in increasing of serum CoQ10, but it is not clear if it relieves myopatic symptoms in statin treated patients. Yet available intervention studies reported contrasting results and just two of them proved benefit of CoQ10 supplementation. Hence coenzyme Q10 supplementation is not currently recommended for routine use in the prophylaxis of statin toxicity.
\end{abstract}

Keywords: Statins, statin-associated myopathy, CoQ10, ubiquinone.

\section{INTRODUCTION}

Statins or 3-hydroxy-3 methylglutharyl coenzyme A reductase inhibitors competitively inhibit cholesterol production by reducing the synthesis of mevalonate, a critical intermediary in the cholesterol pathway. Statins are well tolerated by many patients, but muscle toxicity, also known as myopathy or myotoxicity, is a feared adverse effect of this class of drug. Myopathy is a general term referring to any disease of muscles and myalgia means muscle pain or weakness without increase in creatinine kinase. The most severe form of myotoxicity is rhabdomyolysis, which can occur in all statins, either in monotherapy or in combination therapy, especially with fibrates. Less common adverse effects of statin therapy are hepatotoxicity, peripheral neuropathy, impaired myocardial contractility and autoimmune

*Address correspondence to this author at the 3rd Department of Medicine, Faculty of Medicine PJ Safarik University and Louis Pasteur Hospital, Trieda SNP 1, 04066 Kosice, Slovakia; Tel: 00421/903619556; Fax: 00421/55 6403861; E-mail: dasa.dudova@gmail.com diseases [1-3]. The mechanisms of development of statin-myopathy are unclear. There are several theories proposed to explain it. The first one says that blockage of cholesterol synthesis through statins causes the reduction of sacrolemmal cholesterol, making the skeletal muscle cells unstable [4]. The second theory is based on the assumption that inhibition of small guanosine triphosphate-bindings proteins formation, important to promote cell maintenance and growth and at the same time diminishes apoptosis [4]. Finally, the depletion of CoQ10 in muscle mitochondria during the statin therapy is to increase muscle cell fluidity and thus possibly cause myopathy $[4,5]$. Poor coenzyme Q10 status and antioxidant status in the body are also predisposing factors for statin toxicity [6-10]. A number of observations suggest that statin toxicity may be due to the metabolic effects of lipid lowering in patients with minor muscle disorders [11-13]. These patients have a high frequency of mutations for metabolic muscle diseases and often have depleted mitochondrial enzymes. Their exercise physiology and biopsy findings indicate reduced oxidation of 
fats and mitochondrial dysfunction. These subjects are often intolerant of other lipid lowering therapies in addition to statins, which suggests that the myopathy is due to lipidlowering itself more than a simple pharmacokinetic reaction to high statin levels. Altogether, these findings support the concept that statin myopathy is a metabolic muscle disease [14].

\section{EPIDEMIOLOGY AND RISK FACTORS OF STATIN MYOPATHY}

In randomized clinical trials (RCTs), statin myopathy incidence is about $1.5 \%$ to $5.0 \%[15,16]$. However, incidence of statin myopathy in clinical trials seems to be lower that in real world clinical practice. In clinical trials patients with risk factors of statin toxicity, history of statin related intolerance or developed biochemical abnormalities during the screening period before randomization are usually excluded. In addition, persons who have had previous statin intolerance would probably not enroll in clinical trials, whereas motivated enrolled patients might minimize reporting of mild statin-related myalgias [17]. Several observational studies have documented a $5 \%$ to $10 \%$ incidence of statin-associated myalgia $[18,19]$. The large, observational PRIMO (Prediction of Muscular Risk in Observational Conditions) study [18] of 7924 patients exposed to high-dose statins found that $10.5 \%$ had muscle-related symptoms over 12 months. One observational study of 32225 patients reported that $5.8 \%$ and $6.7 \%$ of diabetic and nondiabetic patients, respectively, had statin-related myalgia [19].

Postmarketing surveillance through the FDA Adverse Event Reporting System (AERS) has documented low reporting rates of statin-related myopathy, myositis, and rhabdomyolysis. From 1998 to 2000, reporting rates for all statins except cerivastatin were $0.38,0.43$, and 1.07 cases per 1 million prescriptions, respectively. From 2002 to 2004, these rates increased to $0.74,0.57$, and 3.56 cases per 1 million prescriptions, respectively, probably because of heightened awareness after the withdrawal of cerivastatin in 2001 [20]. From 2002 to 2004, the FDA AERS rates for myopathy were lowest for fluvastatin ( 0.43 cases per 1 million prescriptions) and highest for rosuvastatin (2.23 cases); for myositis, rates were lowest for atorvastatin ( 0.27 cases) and highest for rosuvastatin (2.37 cases); and for rhabdomyolysis, rates were lowest for pravastatin (1.63 cases) and highest for rosuvastatin (13.54 cases) [20]. The high AERS rates for rosuvastatin, which is the only statin launched after cerivastatin's withdrawal, were attributed to a biased "new drug" reporting effect and to widespread lay media coverage in 2004 [20]. Of importance, the proportionate AERS rate for rosuvastatin was about the same as, or was lower than, that for all statins [20]. Limitations of FDA-derived data on statin myopathy include reliance on voluntary reporting and diagnostic criteria for myopathy or rhabdomyolysis, which required much higher creatine kinase elevations than the ACC/AHA/ NHLBI clinical advisory and perhaps caused underestimation of myopathy incidence [17].

The 2001 AERS rates of fatal rhabdomyolysis varied by agent ( 1 reported case per 5.2 million prescriptions for lovastatin, 23.4 million prescriptions for atorvastatin, 27.1 million prescriptions for pravastatin, and 8.3 million for simvastatin). These low rates starkly contrast with the rate of 1 re- ported case of fatal rhabdomyolysis per about 316000 prescriptions for cerivastatin. No case of fatal rhabdomyolysis has been reported yet with fluvastatin [21]. Thus, although rates of myalgia are higher in clinical practice than in clinical trials and the AERS, the rates of rhabdomyolysis are still reassuringly low (about 0.1 to 0.2 case per 1000 personyears) and are similar to those reported in clinical trials [22].

Higher risk patients for statin myopathy are those with advanced age, females, with a small body frame and frailty, on higher statin doses, on other medications, or with other systemic diseases including hepatic or renal diseases, diabetes mellitus, or hypothyroidism [14]. For example, thin elderly women may represent a demographic category with increased risk for creatine kinase elevations greater than 10 times the upper limit of normal with statin therapy because $5 \%$ to $7 \%$ of women who received cerivastatin, 0.4 to 0.8 $\mathrm{mg} / \mathrm{d}$, had these creatine kinase elevations [23]. Also, only 6 of 22 professional athletes with familial hypercholesterolemia who received statins could tolerate 1 of several statins attempted, indicating that intense physical activity might be a risk factor [24]. During hospitalization for major surgery, the ACC/AHA/NHLBI advises short-term cessation of statin therapy to minimize myopathy risk during the perioperative period [13].

Poor coenzyme Q10 status and antioxidant status in the body are also predisposing factors for statin toxicity [6-10]. One of the most important factors for predicting risk of myopathy is also way of metabolization of statins. Most of them are metabolized by cytochrome P 450 family with the exception of pravastatin [25-27]. Because lovastatin, simvastatin and atorvastatin are metabolized by cytochrome P4503A4 (CYP4503A4), inhibitors of CYP3A4 like cyclosporine, nifedipine, felodipine, amiodarone, fibrates could theoretically increase serum statin levels and exposure to susceptible tissues [28]. Protease inhibitors are potent CYP3A4 inhibitors and thus can increase up to 30 times plasma concentration of statins [29, 30] Cyclosporine is a potent inhibitor of not only CYP4503A4 but also several membrane transporters and it was found to cause many reported cases of rhabdomyolysis [31]. Because pravastatin undergoes renal metabolism, fluvastatin and rosuvastatin are primarily metabolized by CYP2C9, there 3 statins may have a lower myopathy risk, especially in the context of polypharmacy.

Genetic predisposition to statin myopathy is a rapidly expanding area of investigation. Genes of interest include those involved in the pharmacokinetics of the statin response, muscle atrophy, exercise intolerance, pain perception, and mitochondrial energy metabolism [14]. Common DNA polymorphisms in genes encoding cytochrome P450 enzymes, intestinal P-glycoproteins, and organic aniontransporting polypeptide are inconsistently associated with statin myopathy [32-37]. DNA polymorphisms of genes involved in metabolism of coenzyme Q10 and serotonin pain receptors were also inconsistently associated with statin myopathy $[38,39]$. Recently, a common DNA polymorphism in the SLCO1B1 gene encoding organic anion-transporting polypeptide was strongly associated with simvastatinassociated myopathy [32], but this association was not seen in patients with atorvastatin associated myopathy [35]. Finally, among 110 patients with statin myopathy, about $10 \%$ had heterozygous mutations in 1 of several genes that 
normally cause rare myopathy syndromes [40], suggesting that genetic susceptibility to statin myopathy may comprise a complex mixture of rare DNA variants and common DNA polymorphisms.

\section{COQ10}

CoQ10 is naturally occurring, oil-soluble substance, localized in the cell membranes. Around half of ubiquinone is obtained though dietary fat ingestion, while remaining half though endogenous synthesis [41]. Synthesis of CoQ10 in human body decreases with age. CoQ10 is an antioxidant, participating in mitochondrial electron transport during oxidative phoshorylation in mitochondria, transfering electrons from complex I and complex II to complex III of respiratory chain. It forms a very effective redox system, composed of ubiquinole (reduced form), semiquinone radical and ubiquinone (oxidized form). Its reduced form is important scavenger of free oxygen radicals, prevents low-density lipoprotein cholesterol (LDL-C) oxidation and protects membrane lipids and proteins and deoxyribonucleic acid (DNA) against oxidative damage [42]. It also regenerates active forms of the antioxidants ascorbic acid and tocopherol (vitamin E) [43, 44]. In 1980s, it appeared a theory that blocking of farnesyl pyrophosphate resulting in CoQ10 depletion is involved in pathogenesis of statin-associated myopathy [45, 46]. Statins, by reductions of CoQ10 synthesis, can decrease natural antioxidant protection present in our body and predispose toxicity [1-3, 47-51]. The antioxidant status of our body depends on the presence of coenzyme Q10, vitamin A, E, and C and beta- carotine, superoxide dismutase, catalase and ceruloplasmin. Apart from these antioxidants, flavonoids, anthrocyclins, minerals; selenium, chromium and copper and w-3 fatty acids may also provide protection against oxidative stress predisposed by statins [6-8]. It also has been shown that increased levels of CoQ10 may protect cells from chemotherapy-induced oxidative stress [52]. It is suggested that prior or simultaneous administration of antioxidants may be protective against $\mathrm{P} 450$ induced cell damage by the statins, resulting in decrease of statin toxicity [14].

CoQ10 deficiency has been observed in patients with Parkinsons disease, Huntington,s disease, tuberous sclerosis, motor neuron disease, and cerebellar ataxia, CoQ10 supplementation may be useful in these conditions [53-56]. Low levels of CoQ10 have been described also in myocardial biopsies from patients with various cardiovascular diseases $[57,58]$. Lower than normal levels of CoQ10 have been also described in the blood from patients with cardiovascular diseases as compared with levels with healthy human subjects $[59,60]$.

\section{STATINS AND COQ10 IN SERUM, MUSCLES AND MITOCHONDRIA}

The meta-analysis of several clinical trials demonstrates that statin treatment decreases serum CoQ10 levels [61] and in some of the reports the decrease was above $50 \%[10,41$, 45]. Only a few small studies did not confirm this evidence, which is to be explained by the small number of participants and low statin dose $[62,63]$. In the plasma, CoQ 10 is transported with the very low density lipoprotein (VLDL) fraction hence, its levels are closely related to the plasma VLDL levels which can be affected by both the disease processes and by dietary factors. Moreover, whereas the cellular level of
CoQ10 is dependent mostly on endogenous biosynthesis [64], the plasma level has been suggested as representing the equilibrium between $\mathrm{CoQ}_{10}$ absorption and synthesis [65]. There are also some indications, considered contradictory that statin treatment may affect muscle CoQ10 levels. Resulting from the recent human studies the effect of statins may be drug and dose dependent [61] as the significant decrease of muscle CoQ10 levels was found only in the patients treated with simvastatin $80 \mathrm{mg}$ daily [66]. Taken together, it appears that the plasma level of CoQ10 cannot always be regarded as a true indicator of cellular levels. Therefore, in studies in which the cellular levels of CoQ 10 and its changes under pathological conditions are investigated, tissues other than serum or plasma are more desirable [67], but only a few works have examined the intramuscular levels of CoQ10 in patients, who suffered from statin myopathy. In patients with statin associated muscle symptoms or high serum creatinine kinase or both, the muscle biopsies were obtained from 18 patients. No evidence of myocyte apoptosis was found, but only 11 biopsies provided enough tissue to perform the test. The results on muscle CoQ10 levels were controversial, not showing significant CoQ10 level decrease [68].

There should be also an evidence of impaired mitochondrial function, if CoQ10 depletion mediates the statin related myopathy. The data from some animal studies showed structural and functional mitochondria damage in statin treated animals [69-72], while few studies have directly (muscle biopsy) or indirectly (measurement of lactate:pyruvate ratio) addressed this issue in humans. In some studies an increased lactate/pyruvate ratio was found after treatment with statins [5, 73-75], which is used to show a shift toward anaerobic metabolism and could be a possible marker of mitochondrial dysfunction, but the results gained from the other studies did not confirm this observation. In the recent case study, lactic acidosis occurred and was considered as side effect of simvastatin treatment and argues that mitochondrial dysfunction is a cause [76].

\section{COQ10 SUPPLEMENTATION IN STATIN MYOPATHY}

To prevent side effects of statins including myopathy, it should be useful to combine statins with coenzyme Q10; however, greater clinical studies are needed. In a randomized, controlled intervention trial, among 71 patients (Intervention group) and 73 patients (Control group) of acute myocardial infarction (AMI), who were administered coenzyme Q10 (120mg/day) or B vitamins for one year, half of the patients ( $n=36$ vs 31 ) in both groups, received lovastatin (10-20mg/day) [56]. Adverse effects, such as nausea (30,1 vs $9,8 \%)$, vomiting $(13,7$ vs $11,2 \%)$, were more common in the coenzyme Q group whereas fatigue $(6,8$ vs $40,8 \%, \mathrm{p}<0,01)$ was more common in the control group [56]. Fatigue is an early manifestation of muscle damage, indicating that coenzyme Q may have prevented this adverse effect of lovastatin in the intervention group. Initial attempts in administering CoQ10 to statin treated patients with myopathic symptoms had a positive outcome; pain severity significantly decreased after 30 days of CoQ10 supplementation therapy [77, 78].

In 32 hypercholesterolemic patients with statin related myalgia, treatment with CoQ10 (100 mg daily) after 30 days, decreased the intensity of pain by $40 \%$ in the group using 
coenzyme Q10 supplements. In contrast, no change in pain intensity was observed in the group using vitamin E supplements (400 IU/daily) at the end of the trial. Additionally, a decrease of $38 \%$ in pain interference with daily activities was found in CoQ10 group in contrast with no difference in pain interference in the vitamin E group [78]. The pain was assessed using Brief Pain Inventory, which provided measures of pain severity and interference with daily activities [79]. In the randomized, double-blind, placebo controlled study of Mabuchi et al. [80], low dosage of atorvastatin (10 mg/day) has never produced definite liver or muscle damages, and supplementation of CoQ10 (100 mg daily over 16 weeks) has never produced positive results compared with placebo. A more recent trial randomized 44 patients to CoQ10 200 mg daily or placebo for 12 weeks in combination with simvastatin [81]. All patients discontinued lipid-lowering therapy (except ezetimib) and instead started simvastatin dose 10 mg daily, which was uptitrated every 4 weeks towards maximum tolerated dose of $40 \mathrm{mg}$, if was possible. Plasma levels increased in CoQ10 group, but there was no difference in myalgia score or in statin tolerance between both groups. Pain was assessed by using visual analogue scale [82]. The benefit of CoQ10 supplementation was not shown in this trial due to a several limitations: patients did not experience sufficiently severe myalgias, the absorption of CoQ10 likely to be decreased as the patients were initiated CoQ10 at the same time as the statin therapy. More recently, benefit of CoQ10 supplementation was shown in another randomized, placebo contolled trial (currently only available in abstract), using CoQ10 $200 \mathrm{mg}$ daily during 3 months in patients with statin associated myopathy defined as myalgia, fatigue, muscle cramps and muscle weaknes [83]. The present evidence does not support routine coenzyme Q10 supplementation in statin-associated myopathy. However, guidelines of some associations recommend using CoQ10 supplements in patients with the risk factors of statin-associated myopathy [84]. Possible risks of widely prescribed statin therapy should be decreased by administering of lower doses, combination with coenzyme Q10 and avoidance of risky combinations of drugs with interfering metabolism via cytochrome $\mathrm{P}$ 450. The role of lipid lowering diet plus exercise is indisputable and may support lower doses of lipid lowering drugs or even lower use of these drugs [85] and may prevent prooxidative stage that is important for statin intolerance. Use of coenzyme Q10 even in smaller doses of $30 \mathrm{mg} / \mathrm{day}$ may be rewarding in the prevention of adverse effects of statins $[56,86]$.

\section{CONCLUSIONS}

Myalgia is the most common adverse event of statins leading to nonadherence or even stopping of statin therapy. It affects up to $10 \%$ of patients receiving statin treatment, statin-induced fatal rhabdomyolysis is extremely rare. It is plausible that statin block the production of mevalonate, which is the precursor of CoQ10. Statin interference with CoQ10 production prompted the hypothesis that pathogenesis of statin myopathy may be related in part to statin inhibition of the endogenous synthesis of coenzyme Q10.

However, coenzyme Q10 supplementation is not currently recommended for routine use. There is at least a need to consider coenzyme Q10 administration in conjunction with statin when they are given $>20 \mathrm{mg} /$ day, as it might also prevent deaths due to myopathy and other toxic effects because statin toxicity is considered a metabolic myopathy [27, 87].

There are not known risks for CoQ10 supplementation in mild to moderate dosage (30-300 mg daily), which may support its usage in common medical practice in patients with statin related myopathy. It is postulated, that effect of CoQ10 supplementation is dose and form dependent; the therapeutic response likely to correlate with CoQ10 plasma levels achieved through supplementation therapy (target treatment levels 2,5-3,5 $\mu \mathrm{mol} / \mathrm{L})$. Therefore, when CoQ10 supplementation is occurring, it is advisable to monitor the CoQ10 levels to ensure the efficacy, as there a variable bioavailability of commercial formulations and inter-individual variation in CoQ10 absorption exist. CoQ10 is available in several forms, including powder, suspension, oil solution, solubilized forms (All Q and Q-gel), all with different bioavailability. Using a lipid formulations and taking CoQ10 with food improves absorption [88].

Coenzyme Q10 supplementation may offer an alternative to stopping treatment with these drugs, if adverse effects appear. This may be of great importance, as the statins represent the basic pillars for primary and secondary prevention of atherosclerosis and their prescription rate is increasing dramatically.

\section{REFERENCES}

[1] Radcliffe KA, Campbell WW. Statin myopathy. Curr Neurol Neurosci Rep 2008; 8: 66-72.

[2] Venero CV, Thompson PD. Managing statin myopathy. Endocrinol Metab Clin North Am 2009; 38: 121-36.

[3] Phillips PS, Haas RH. Statin myopathy as a metabolic muscle disease. Expert Rev Cardiovasc Ther 2008; 6: 971-8.

[4] Thompson PD, Clarkson P, Karas RH. Statin-associated myopathy. JAMA 2003; 289: 1681-90.

[5] Phillips PS, Haas RH, Bannykh S. Statin-associated myopathy with normal creatine kinase levels. Ann Intern Med 2002; 137: 581-5.

[6] Koumis T, Nathan JP, Rosenberg JM, Cicero LA. Strategies for the prevention and treatment of statin-induced myopathy: is there a role for ubiquinone supplementation? Am Health Syst Pharmacol 2004; 61: 515-9.

[7] Chong P, Seeger J, Franklin C. Clinically relevant differences between the statins: implication for therapeutic selection. Am J Med 2001; 111: 390-400.

[8] Bliznakov EG. Lipid lowering drugs (statins), cholesterol and coenzyme Q10 - The Baycol case-a modern Pandora's box. Biomed Pharmacother 2002; 56: 56-9.

[9] Mortensen SA, Leth A, Agner E, Rohde M. Dose- related decrease of serum coenzyme Q10 during treatment with HMG-CoA reductase inhibitors. Mol Aspects Med 1997; 18(Suppl S): S137-44.

[10] Rundek T, Naini A, Sacco R, Coates K, DiMauro S. Atorvastatin decreases the coenzyme Q10 level in the blood of patients at risk for cardiovascular disease and stroke. Arch Neurol 2004; 61: 889-92.

[11] Joint National Committee on Detection, Evaluation, and Treatment of High Blood Pressure. The 1984 report of the Joint National Committee on Detection, Evaluation, and Treatment of High Blood Pressure. Arch Intern Med 1984; 144: 1045-57.

[12] Pella D, Rybar R, Valocik G, Petrasová D, Trejbal D, Mitro P. Secondary prevention of atherosclerosis by combined treatment with sulodexide and fluvastatin. Cardiovascular Diseases. Bologna: Monduzzi Editore 2002; pp. 289-93.

[13] Pasternak RC, Smith SC Jr, Bairey-Merz CN, Grundy SM, Cleeman JI, Lenfant C; American College of Cardiology. ACC/AHA/ NHLBI clinical advisory on the use and safety of statins. J Am Coll Cardiol 2002; 40: 567-72.

[14] Fedacko J, Singh RB, Chaithirapan S, et al. Clinical manifestation of adverse events of statins, oxidative stress and possible role of antioxidants in prevention? Open Nutra J 2010; 3: 154-65. 
[15] Bays H. Statin safety: an overview and assessment of the data2005. Am J Cardiol 2006; 97: 6C-26C.

[16] Law M, Rudnicka AR. Statin safety: a systematic review. Am J Cardiol 2006; 97: 52C-60C.

[17] Joy TR, Hegele RA. Narrative review: statin-related myopathy. Ann Intern Med 2009; 150(12): 858-68

[18] Bruckert E, Hayem G, Dejager S, Yau C, Be'gaud B. Mild to moderate muscular symptoms with high-dosage statin therapy in hyperlipidemic patients-the PRIMO study. Cardiovasc Drugs Ther 2005; 19: 403-14.

[19] Nichols GA, Koro CE. Does statin therapy initiation increase the risk for myopathy? An observational study of 32,225 diabetic and nondiabetic patients. Clin Ther 2007; 29: 1761-70.

[20] Davidson MH, Clark JA, Glass LM, Kanumalla A. Statin safety: an appraisal from the adverse event reporting system. Am J Cardiol 2006; 97: 32C-43C.

[21] Staffa JA, Chang J, Green L. Cerivastatin and reports of fatal rhabdomyolysis [Letter]. N Engl J Med 2002; 346: 539-40.

[22] Silva MA, Swanson AC, Gandhi PJ, Tataronis GR. Statin-related adverse events: a meta-analysis. Clin Ther 2006; 28: 26-35.

[23] Jacobson TA. Statin safety: lessons from new drug applications for marketed statins. Am J Cardiol 2006; 97: 44C-51C

[24] Sinzinger H, O'Grady J. Professional athletes suffering from familial hypercholesterolaemia rarely tolerate statin treatment because of muscular problems. Br J Clin Pharmacol 2004; 57: 525-8.

[25] Nelson DR, Katamaki T, Waxman DL, et al. The P450 superfamily: update on new sequences, gene mapping, accession numbers, early trivial names of enzymes and nomenclature. DNA Cell Biol 1993; 12: $1-51$.

[26] Nebert DW. The P450 superfamily: update on new sequences, gene mapping and recommended nomenclature. DNA Cell Biol 1991; 10: 1-14.

[27] London SF, Gross KF, Ringel SP. Cholesterol lowering agent myopathy. Neurology 1991; 41: 1159-60.

[28] Baker SK, Samjoo IA. A neuromuscular approach to statinrelated myotoxicity. Can J Neurol Sci 2008; 35:8-21.

[29] Ray GM. Antiretroviral and statin drug-drug interactions. Cardiol Rev 2009; 17: 44-7

[30] Fichtenbaum CJ, Gerber JG, Rosenkranz SL, et al.; NIAID AIDS Clinical Trials Group. Pharmacokinetic interactions between protease inhibitors and statins in HIV seronegative volunteers: ACTG Study A5047. AIDS 2002; 16: 569-77.

[31] Neuvonen PJ, Niemi M, Backman JT. Drug interactions with lipidlowering drugs: mechanisms and clinical relevance. Clin Pharmacol Ther 2006; 80: 565-81.

[32] SEARCH Collaborative Group. SLCO1B1 variants and statininduced myopathy - a genomewide study. N Engl J Med 2008; 359: 789-99.

[33] Fiegenbaum M, da Silveira FR, Van der Sand CR, et al. The role of common variants of ABCB1, CYP3A4, and CYP3A5 genes in lipid-lowering efficacy and safety of simvastatin treatment. Clin Pharmacol Ther 2005; 78: 551-8.

[34] Frudakis TN, Thomas MJ, Ginjupalli SN, et al. CYP2D6*4 polymorphism is associated with statin-induced muscle effects. Pharmacogenet Genomics 2007; 17: 695-707.

[35] Hermann M, Bogsrud MP, Molden E, et al. Exposure of atorvastatin is unchanged but lactone and acid metabolites are increased several-fold in patients with atorvastatin-induced myopathy. Clin Pharmacol Ther 2006; 79: 532-9.

[36] Mulder AB, van Lijf HJ, Bon MA, et al. Association of polymorphism in the cytochrome CYP2D6 and the efficacy and tolerability of simvastatin. Clin Pharmacol Ther 2001; 70: 546-51.

[37] Zuccaro P, Mombelli G, Calabresi L, Baldassarre D, Palmi I, Sirtori CR. Tolerability of statins is not linked to CYP450 polymorphisms, but reduced CYP2D6 metabolism improves cholesteraemic response to simvastatin and fluvastatin. Pharmacol Res 2007; 55: 310-7.

[38] Oh J, Ban MR, Miskie BA, Pollex RL, Hegele RA. Genetic determinants of statin intolerance. Lipids Health Dis 2007; 6: 7.

[39] Ruaño G, Thompson PD, Windemuth A, et al. Physiogenomic association of statin-related myalgia to serotonin receptors. Muscle Nerve 2007; 36: 329-35.

[40] Vladutiu GD, Simmons Z, Isackson PJ, et al. Genetic risk factors associated with lipid-lowering drug-induced myopathies. Muscle Nerve 2006; 34: 153-62.
[41] Ghirlanda G, Oradei A, Manto A. Evidence of plasma CoQ10 lowering effect by HMG-CoA reductase inhibitors: a double blind, placebo-controlled study. J Clin Pharmacol 1993; 33: 226-9.

[42] James AM, Smith RA, Murphy MP. Antioxidant and prooxidant properties of mitochondrial coenzyme Q. Arch Biochem Biophys 2004; 423: 47-56.

[43] Arroyo A, Navarro F, Gomez-Diaz C. Interactions between ascorbyl free radical and coenzyme Q at the plasma membrane. J Bioenerg Biomembr 2000; 32: 199-210.

[44] Constantinescu A, Maguire JJ, Packer L. Interactions between ubiquinones and vitamins in membranes and cells. Mol Aspects Med 1994; 15(Suppl): s57-65.

[45] Folkers K, Langsjoen P, Willis R. Lovastatin decreases koenzyme Q levels in humans. Proc Natl Acad Sci U S A 1990; 87: 8931-4.

[46] Walravens PA, Greene C, Frerman FE. Lovastatin, isoprenes, and myopathy. Lancet 1989; 2: 1097-98.

[47] Golomb BA, Kwon EK, Koperski S, Evans MA. Amyotrophic lateral sclerosis-like conditions in possible association with cholesterol-lowering drugs: An Analysis of PatientReports to the University of California, San Diego (UCSD) Statin Effects Study. Drug Saf 2009; 32: 649-61.

[48] Colman E, Szarfman A, Wyeth J, et al. An evaluation of a data mining signal for amyotrophic lateral sclerosis and statins detected in FDA's spontaneous adverse event reporting system. Pharmacoepidemiol Drug Saf 2008; 17: 1068-76.

[49] Fernández AB, Karas RH, Alsheikh-Ali AA, Thompson PD. Statins and interstitial lung disease: a systematic review of the literature and of food and drug administration adverse event reports. Chest 2008; 134: 824-30.

[50] Tuccori M, Lapi F, Testi A, et al. Statin-associated psychiatric adverse events: a case/non-case evaluation of an Italian database of spontaneous adverse drug reaction reporting. Drug Saf 2008; 31: 1115-23.

[51] Fraunfelder FW, Richards AB. Diplopia, blepharoptosis, and ophthalmoplegia and 3-hydroxy-3-methyl-glutaryl-CoA reductase inhibitor use. Ophthalmology 2008; 115: 2282-5.

[52] Brea-Calvo G, Rodríguez-Hernández A, Fernández-Ayala DJ, Navas P, Sánchez-Alcázar JA. Chemotherapy induces an increase in coenzyme Q10 levels in cancer cell lines. Free Radic Biol Med 2006; 40(8): 1293-302.

[53] Group HsS. A randomized, placebo controlled trial of coenzyme Q10 ramacemide in Huntington's disease. Neurology 2001; 57: 375-6.

[54] Lamperti C, Naini A, Hirano M, et al. Cerebellar ataxia and coenzyme Q10 deficiency. Neurology 2003; 60(7): 1206-8.

[55] Naini A, Lewis VJ, Hirano M, et al. Primary coenzyme Q10 deficiency and the brain. Biofactors 2003; 18(1-4): 145-52.

[56] Singh RB, Neki NS, Kartikey K, et al. Effect of coenzyme Q10 on risk of atherosclerosis in patients with recent myocardial infarction. Mol Cell Biochem 2003; 246(1-2): 75-82.

[57] Littaru GP, Ho L, Folkers K. Deficiency of CoQ10 in human heart disease. II Int J Vitam Nutr Res 1972; 42: 291-5.

[58] Folkers K, Litarru GP, Ho L, et al. Evidence for deficiency of CoQ10 in human heart disease. Int Vit Nutr Res 1970; 40: 380 90

[59] Folkers K, Baker L, Richardson, et al. Biomedical and clinical aspects of CoQ10, In: Yamamura Y, Folkers K, Ito Y. Eds. Amsterdam, Elsevier, 1980; Vol 2, p. 449

[60] Mortensen SA. Perspective of therapy of cardiovascular diseases with CoQ10 (ubiquinone). Clin Invest 1993; 71(8 Suppl): S11623.

[61] Marcoff L, Thompson PD. The role of coenzyme Q10 in statinassociated myopathy: a systematic review. J Am Coll Cardiol 2007 49: 2231-7.

[62] Miyake Y, Shouzu A, Nishikawa M. Effect of treatment with 3hydroxy-3-methylglutaryl coenzyme A reductase inhibitors on serum coenzyme Q10 in diabetic patients. Arzneimittelforschung 1999; 49: 324-9.

[63] Bleske BE, Willis RA, Anthony M. The effect of pravastatin and atorvastatin on coenzyme Q10. Am Heart J 2001; 142: E2.

[64] Ernster L, Dallner G. Biochemical, physiological and medical aspects of ubiquinone function. Biochim Biophys Acta 1995 1271(1): 195-204.

[65] Folkers K, Wolanivk A. Progress in biomedical research on coenzyme Q10. Drugs Exp Clin Res 1984; 10: 513-517. 
[66] Paiva H, Thelen KM, Van Coster R. High-dose statins and skeletal muscle metabolism in humans: a randomized, controlled trial. Clin Pharmacol Ther 2005; 78: 60-68.

[67] Singh RB, Naini A. Cerebrospinal Fluid (CSF) concentration of CoQ10 in clinical practice, breaking news finding in humans. Open Nutra J 2009; 2: 13-5.

[68] Lamperti C, Naini AB, Lucchini V. Muscle coenzyme Q10 level in statin-related myopathy. Arch Neurol 2005; 62: 1709-12.

[69] Schaefer WH, Lawrence JW, Loughlin AF. Evaluation of ubiquinone concentration and mitochondrial function relative to cerivastatin-induced skeletal myopathy in rats. Toxicol Appl Pharmacol 2004; 194: 10-23.

[70] Diebold BA, Bhagavan NV, Guillory RJ. Influences of lovastatin administration on the respiratory burst of leukocytes and the phosphorilation potential of mitochondria in guinea pigs. Biochim Biophys Acta 1994; 1200: 100-8.

[71] Satoh K, Yamato A, Nakai T. Effects of 3-hydroxy-3methylglutharyl ceonzyme A reductase inhibitors on mitochondrial respiration in ischaemic dog hearts. Br J Pharmacol 1995; 116: 1894-8.

[72] Sugiyama S. HMG CoA reductase inhibitor accelerates aging effect on diaphragm mitochondrial respiratory function in rats. Biochem Mol Biol Int 1998; 46: 923-31.

[73] Phillips PS, Haas RH, Bannykh S. Statin-associated myopathy with normal creatinine kinase levels. Ann Intern Med 2002; 137: 581-5.

[74] Phillips PS, Phillips CT, Sullivan MJ. Statin myotoxicity is associated with changes in cardiopulmonary function. Atherosclerosis 2004; 177: 183-8.

[75] Paoliso G, Barbagallo M, Petrella G. Effects of simvastatin and atorvastatin administration on insulin resistance and respiratory quotient in aged dyslipidemic non insulin dependent diabetic patients. Atherosclerosis 2000; 150: 121-7.

[76] Goli AK, Goli SA, Byrd RP Jr, Roy TM. Simvastatin-induced lactic acidosis: a rare adverse reaction? Clin Pharmacol Ther 2002; 72: 461-4.

[77] Chapidze G E, Kapanadze SD, Dolidze NK. Combination treatment with CoQ10 and simvastatin in patients with coronary atherosclerosis (Russian). Kardiologiia 2006; 46(8): 11-3.
[78] Caso G, Kelly P, McNurlan MA. Effect of coenzyme Q10 on myopathic symptoms in patients treated with statins. Am J Cardiol 2007; 99: 1409-12.

[79] Keller S, Bann CM, Dodd SL, Schein J, Mendoza TR, Cleeland CS. Validity of the brief pain inventory for use in documenting the outcomes of patients with noncancer pain. Clin J Pain 2004; 20 : 309-18.

[80] Mabuchi H, Nohara A, Kobayashi J, et al. Effects of CoQ10 supplementation on plasma lipoprotein lipid, CoQ10 and liver and musce enzyme levels in hypercholesterolemic patients treated with atorvastatin: a randomized, double-blind study. Atherosclerosis 2007; 195:e182-189.

[81] Young JM, Florkowski CM, Molyneux SL, et al. Effect of CoQ10 on Simvastatin-Induced Myalgia. Am J Cardiol 2007; 100: 14003 .

[82] Landstad BJ, Schuldt K, Ekholm J, Broman L, Bergroth A. Women at work despite ill health: diagnoses and pain before and after personnel support. A prospective study of hospital cleaners/home-help personnel with comparison groups. J Rehabil Med 2001; 33: 21624.

[83] Fedacko J, Pella D, Rybar R, et al. Coenzyme Q10 and selenium in statin-associated myopathy treatment. Results of randomized double-blind clinical study. Eur Heart J (abstr) 2009; 234.

[84] European guidelines on cardiovascular disease prevention in clinical practice: executive summary (Slovak). Cardiology 2008; 17(Suppl. 3): 2S-36S.

[85] Singh RB, Dubnov O, Niaz MA, et al. The effect of an indomediterranean diet on the progression of coronary artery disease in high risk patients. The Indo-Mediterranean Diet Heart Study. Lancet 2002; 360: 1455-61.

[86] Chiang CA, Pella D, Singh RB. Adverse effects of hmg coareductase inhibitors and the role of coenzyme q10. J Nutr Environ Med 2004; 14: 17-28.

[87] Golomb BA, Evans MA. Statin adverse effects: a review of the literature and evidence for a mitochondrial mechanism. Am J Cardiovasc Drugs 2008; 8: 373-418.

[88] Beal MF, Shuts CW. Effects of CoQ10 in Huntington's disease and early Parkinson disease. Biofactors 2003; 18(1-4): 153-61.

C Skripova et al.; Licensee Bentham Open.

This is an open access article licensed under the terms of the Creative Commons Attribution Non-Commercial License (http://creativecommons.org/licenses/by-nc/3.0/) which permits unrestricted, non-commercial use, distribution and reproduction in any medium, provided the work is properly cited. 